\title{
Relationship between adverse events associated with lenvatinib treatment for thyroid cancer and patient prognosis
}

\author{
HIROYUKI IWASAKI, SOJI TODA, DAISUKE MURAYAMA, SHIN KATO and AI MATSUI \\ Department of Breast and Endocrine Surgery, Kanagawa Cancer Center, Yokohama, Kanagawa 241-8515, Japan
}

Received July 2, 2020; Accepted November 3, 2020

DOI: $10.3892 / \operatorname{mco} .2020 .2190$

\begin{abstract}
Tyrosine kinase inhibitors (TKIs) were first approved for treating radioactive iodine-refractory differentiated thyroid cancer (DTC) and anaplastic thyroid cancer (ATC) 5 years ago. Among them, lenvatinib has a high response rate and has become the first-line drug for treating thyroid cancer. Although it has a high response rate, it is also characterized by a high frequency of adverse events (AEs). AEs previously reported in a phase II study occurred after the practical application of TKI therapy. However, the type and frequency of AEs that occurred were significantly different. The present study investigated the type and frequency of AEs in the real-world setting and examined their relationship with prognosis. Between June 2015 and May 2020, 111 patients (79 patients with DTC and 32 patients with ATC) were treated with lenvatinib. An investigation of lenvatinib AEs, including fatal events, revealed that fistula formation or severe tumor regrowth after the discontinuation of treatment was an $\mathrm{AE}$ associated with poor prognosis. In total, 11 patients with ATC (34.4\%) and 7 patients with DTC (8.9\%) developed skin fistula. The mortality rate among these patients was $38.9 \%$ (7/18), including three deaths caused by major bleeding and four deaths attributable to mediastinitis or pneumonia. In the DTC group, irreversible regrowth occurred in all 7 patients who required drug withdrawal because of AEs, and all patients died. Conversely, overall survival was longer among patients with DTC and hand-foot syndrome (HFS). Therefore, the present study illustrated that although lenvatinib therapy can result in severe AEs requiring dose reduction or treatment discontinuation, the HFS appearance portends a good prognosis in patients treated with lenvatinib.
\end{abstract}

Correspondence to: Dr Hiroyuki Iwasaki, Department of Breast and Endocrine Surgery, Kanagawa Cancer Center, 2-3-2 Nakao, Asahi-ku, Yokohama, Kanagawa 241-8515, Japan

E-mail: iwasaki.h@kcch.jp

Abbreviations: TKI, tyrosine kinase inhibitor; DTC, differentiated thyroid cancer; ATC, anaplastic thyroid cancer; AE, adverse event; HFS, hand-foot syndrome; OS, overall survival

Key words: thyroid cancer, lenvatinib, adverse event, hand-foot syndrome, retrospective study, prognosis

\section{Introduction}

The age-adjusted incidence and mortality of thyroid cancer was 15.03 and 0.51 per 100,000 individuals, respectively, in 2015 (https://seer.cnacer.gov/csr/1975_2115/). The increasing incidence of thyroid cancer is mostly because of increasing surveillance and overdiagnosis $(1,2)$.

In Japan, the age-adjusted incidence of thyroid cancer was 8.7 per 100,000 person-years in 2017 , whereas the age-adjusted mortality rate was 0.3 per 100,000 person-years in 2018 (https://ganjoho.jp/reg_stat/index.html). Since the introduction of tyrosine kinase inhibitors (TKIs) for cancer treatment, their efficacy and adverse events (AEs) have been reported for various carcinomas $(3,4)$. TKI treatment has also been introduced as a guideline for treating patients with radioactive iodine (RAI)-refractory differentiated thyroid carcinoma (DTC) (5). Patients with recurrent distant metastases of advanced thyroid cancer are refractory to RAI; another option for TKI treatment is external irradiation, but there are limited sites and frequency of this alternative treatment. The therapeutic effect of TKIs depends on the target lesion (6). For instance, the treatment results for pulmonary metastases are excellent, whereas those for bone metastases and unresectable local lesions are poor. The efficacy of TKIs represented a breakthrough for cancer treatment, and these drugs have attracted the attention of several clinicians. However, their clinical utility is limited by frequent AEs $(7,8)$. High blood pressure, cutaneous symptoms, and digestive tract symptoms can be treated with appropriate management. Conversely, tumor necrosis-induced cavitation resulting in macrovascular rupture (9) and rapid tumor regrow th after treatment discontinuation (10) are considered as life-threatening AEs. However, the prognosis of TKIs is favorable despite the appearance of AEs. In particular, the appearance of hand-foot syndrome (HFS) is reportedly correlated with prognosis for several TKIs (11-13). This retrospective study aimed to verify the AEs of lenvatinib in the real-world setting and clarify whether life-threatening AEs and HFS are markers of good prognosis as previously reported.

\section{Materials and methods}

Patients'selection. Between June 2015 and May 2020, 111 patients were treated at Kanagawa Cancer Center, Japan with lenvatinib, including 79 patients with radioactive 
Table I. AEs observed in the DTC and ATC groups.

\begin{tabular}{|c|c|c|c|c|}
\hline \multirow[b]{2}{*}{ AEs } & \multicolumn{2}{|c|}{ DTC $(n=79)$} & \multicolumn{2}{|c|}{$\operatorname{ATC}(n=32)$} \\
\hline & All-grade & Grade $\geq 3$ & All-grade & Grade $\geq 3$ \\
\hline Hypertension, \% & 91.1 & 8.9 & 75.0 & 9.4 \\
\hline Proteinuria, $\%$ & 64.6 & 15.2 & 40.6 & 3.1 \\
\hline Appetite loss, $\%$ & 43.0 & 5.1 & 46.9 & 6.2 \\
\hline HFS, $\%$ & 40.5 & 5.1 & 18.8 & 6.2 \\
\hline Liver dysfunction, \% & 16.5 & 1.3 & 12.5 & 3.1 \\
\hline Fatigue, $\%$ & 15.2 & 0.0 & 12.5 & 3.1 \\
\hline Alopecia, \% & 12.7 & 0.0 & 0.0 & 0.0 \\
\hline Stomatitis, \% & 10.1 & 0.0 & 9.4 & 0.0 \\
\hline Diarrhea, $\%$ & 8.9 & 0.0 & 6.2 & 0.0 \\
\hline \multicolumn{5}{|l|}{ Serious AEs, n (\%) } \\
\hline Skin fistula & \multicolumn{2}{|c|}{$7(8.9)$} & \multicolumn{2}{|c|}{$11(34.4)$} \\
\hline Cavitation & \multicolumn{2}{|c|}{$1(1.3)$} & \multicolumn{2}{|c|}{$2(6.3)$} \\
\hline Embolism & \multicolumn{2}{|c|}{$4(5.1)$} & \multicolumn{2}{|c|}{$2(6.3)$} \\
\hline GI perforation & \multicolumn{2}{|c|}{$2(2.6)$} & \multicolumn{2}{|c|}{$0(0.0)$} \\
\hline Regrowth & \multicolumn{2}{|c|}{$7(8.9)$} & \multicolumn{2}{|c|}{$0(0.0)$} \\
\hline
\end{tabular}

AE, adverse event; DTC, differentiated thyroid cancer; ATC, anaplastic thyroid cancer; HFS, hand-foot syndrome; GI, gastrointestinal.

iodine-refractory DTC and 32 patients with anaplastic thyroid cancer (ATC). This study was approved by the Institutional Review Board of Kanagawa Cancer Center (IRB approval number 27-61 for DTC and 28-49 for ATC). All patients provided a comprehensive consent stating that their samples collected for medical examination could be utilized for investigation and clinical research.

Treatment dose and AE evaluation. The initial dose of lenvatinib was $24 \mathrm{mg} / \mathrm{day}$, and the dose could be reduced for the following reasons: Body weight $<40 \mathrm{~kg}$, age $\geq 80$ years, performance status $\geq 2$, heart failure, and chronic kidney disease. Interruption or reduction of dose (to $20,14,10$ or $8 \mathrm{mg} /$ day) was permitted in cases of toxic effects. The dose reduction criteria for proteinuria were as reported previously (14). We classified the radiologic response to TKI therapy on the basis of the RECIST 1.1 criteria (15) as follows: Complete remission of the disease (CR), partial response (PR), stable disease (SD), and progressive disease (PD). To evaluate safety, the occurrence of any $\mathrm{AE}$ and the time for treatment discontinuation were recorded. AEs were graded from 1 to 5 according to the Common Terminology Criteria for Adverse Events (CTCAE) version 5.0 (http://www.jcog.jp/doctor/tool/ctcaev5.html), and the maximum value was totaled for each patient. Table I shows the frequency of all grades and that of $\geq$ grade 3 . The frequency of $\geq$ grade 3 was low. Grade 4 indicated a life-threatening AE, which included skin fistula, cavitation, embolism, GI perforation, and regrowth, requiring drug discontinuation.

Statistical analysis. The median values between the two groups were compared using the Fisher's test for nominal variables and the Student's t-test for continuous variables. The statistically significant difference was set at $\mathrm{P}<0.05$. All statistical data were analyzed using EZR software version 2.4. (16) Furthermore, survival analysis for DTCs was performed using the Cox hazard model for the AEs listed in Table I. Overall survival (OS) after lenvatinib treatment and 12-month survival rates were calculated using the Kaplan-Meier method with SPSS software (version 24; IBM Corp.), and the log-rank and Bonferroni tests were applied. $\mathrm{P}<0.05$ was considered statistically significant.

\section{Results}

Patient characteristics. Table II presents the patient characteristics of the ATC and DTC groups. Patients with ATC were significantly older, and they had a worse treatment outcome. In addition, patients with ATC had shorter treatment periods and survival.

Frequency and characteristics of AEs. Meanwhile, the frequency of AEs is presented in Table I. The most common AE was hypertension (HT) in both groups, and approximately half of such patients had HT as a pre-existing disease before treatment that was exacerbated by lenvatinib therapy. The second most common AE was proteinuria, followed by anorexia and HFS, while diarrhea occurred less frequently because antidiarrheal drugs were often prescribed at the beginning of treatment. HT and HFS were observed as an early-phase AE in all patients within 1 month following lenvatinib treatments. The frequencies of HFS and proteinuria were higher in patients with DTC who had long treatment periods, whereas alopecia was only observed as a late-phase AE in patients with DTC who used lenvatinib for $>1$ year. Concerning serious AEs, skin fistula appeared in 11 patients with DTC (34.4\%) and 7 patients with ATC (8.9\%). In both groups, fistulae were caused by tumor necrosis in the lesion adjacent to the trachea or skin. One typical case is presented in Fig. 1. The fatality 
Table II. Patient characteristics in the ATC and DTC groups.

\begin{tabular}{lcc}
\hline & \multicolumn{2}{c}{ Group } \\
\cline { 2 - 3 } Parameter & ATC & DTC \\
\hline Number & 32 & 79 \\
Age, years & $77(42-89)$ & $72(41-87)$ \\
Female, n $\%)$ & $18(56.2)$ & $49(62.0)$ \\
Male, n $\%)$ & $14(43.8)$ & $30(38.0)$ \\
Height, cm & $157.2(133.9-174.1)$ & $154.9(135.1-174.0)$ \\
BW, kg & $53.1(41.5-88.0)$ & $55.9(32.7-101.4)$ \\
PR, n $(\%)$ & $6(18.8)$ & $29(36.7)$ \\
SD, n $(\%)$ & $8(25.0)$ & $37(46.8)$ \\
NE, n $(\%)$ & $6(18.8)$ & $0(0.0)$ \\
PD, n $(\%)$ & $12(37.5)$ & $13(16.5)$ \\
OS, months & $3.2(0.5-28.9)$ & $19.9(1.0-62.3)$ \\
Initial dose, mg & $24.0(10-24)$ & $24.0(8-24)$ \\
Duration, months & $3.2(0.2-27.9)$ & $18.2(0.7-62.3)$ \\
\hline
\end{tabular}

${ }^{\mathrm{a}} \mathrm{P}<0.05$ (Student's t-test); ${ }^{\mathrm{b}} \mathrm{P}<0.05$ (Fisher's test). The median values between two groups were compared using Fisher's test for nominal variables and Student's t-test for continuous variables. Continuous variables are shown as the median and range (minimum-maximum). ATC, anaplastic thyroid cancer; DTC, differentiated thyroid cancer; BW, body weight; PR, partial response; SD, stable disease; NE, not evaluable; PD, progressive disease; OS, overall survival.
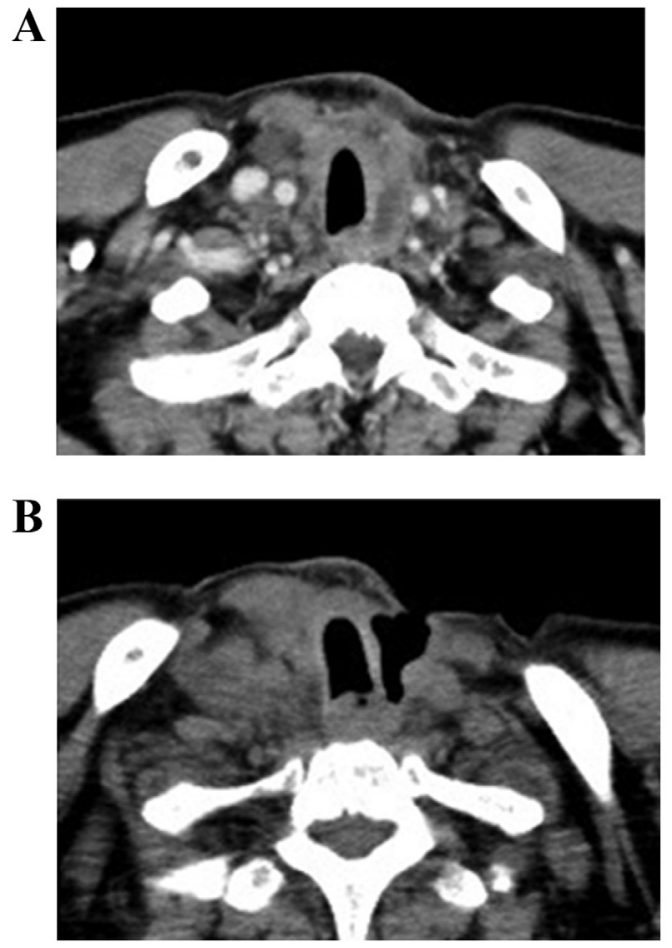

Figure 1. A case of differentiated thyroid cancer involving the appearance of tracheoesophageal cutaneous fistula. (A) Local cervical recurrence before treatment and multiple lung metastases were present. (B) Fistula formation was noted after 12 weeks of treatment with lenvatinib $(20 \mathrm{mg})$, necessitating dose reduction to $10 \mathrm{mg}$. Treatment was discontinued after 6 additional weeks of therapy due to fistula expansion.

rate associated with this $\mathrm{AE}$ was $38.9 \%$ (7/18), including three deaths because of major bleeding and four deaths attributable to successive pneumonia and mediastinitis. Three patients
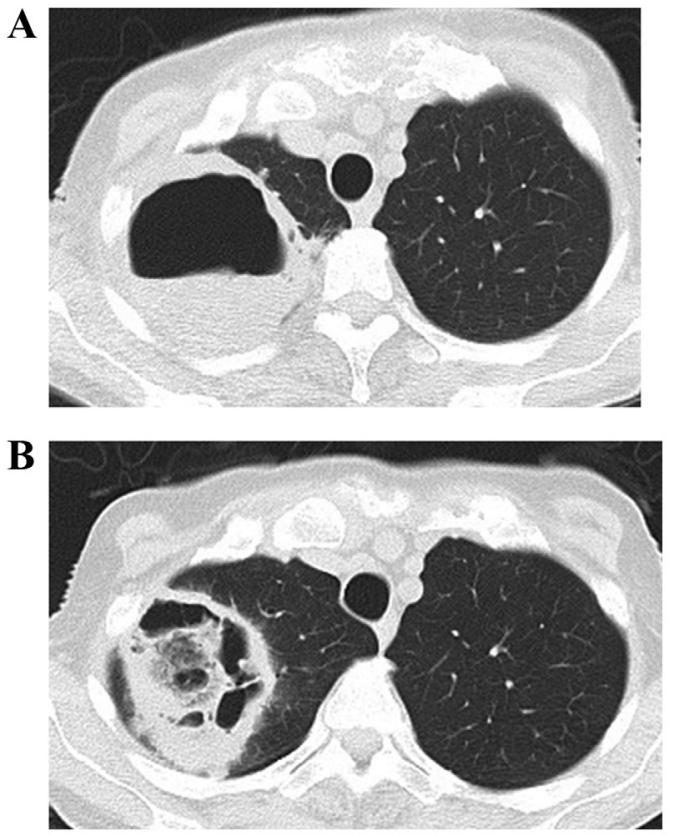

Figure 2. A case of differentiated thyroid cancer involving cavitation in the upper lobe of the right lung. (A) Lenvatinib therapy at $24 \mathrm{mg}$ was started for multiple bone metastases and local recurrence. After 8 weeks, the patient exhibited loss of appetite and malaise, and the dose was reduced to $10 \mathrm{mg}$. The image was captured after 20 weeks. Leachate accumulated in the cavity. In both sputum culture and blood testing, no causative bacteria were noted that could form cavities in the lungs. (B) Image captured 8 weeks after treatment discontinuation. Leachate levels in the cavity had declined, and sputum appeared; however, the size of the cavity was mostly unchanged.

(two patients with ATC and one patient with DTC) displayed cavity formation in the lungs that prevented further treatment with lenvatinib. One particular case is shown in Fig. 2. 
Table III. Characteristics of adverse events of lenvatinib treatment for patients with differentiated thyroid cancer.

\section{Characteristics of AEs}

$\mathrm{AE}$

Early phase AE
(within 1 month)
Late phase $\mathrm{AE}$
(>1 year)
All phase AE

AE with poor prognosis

AE with favorable prognosis

${ }^{\text {aSkin }}$ fistula, cavitation, embolism, GI perforation. AE, adverse event; DTC, differentiated thyroid cancer; HT, hypertension; HFS, hand-foot syndrome.

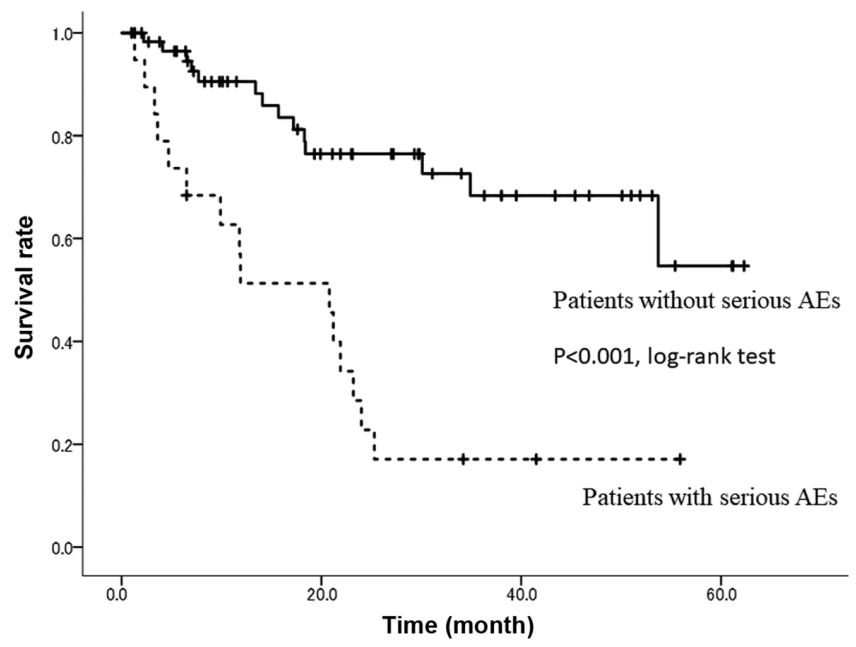

Figure 3. Comparison of OS curves between patients with and without serious AEs. The bold line is the survival curve for patients with DTC without serious AEs. The dotted line is the survival curve for patients with DTC with serious AEs. The survival rate after 24 months of treatment in patients without serious AEs was 76.5\%, compared with $22.8 \%$ for patients with serious AEs. The OS curves were significantly different according to the log-rank test $(\mathrm{P}<0.001)$. AEs, adverse events; DTC, differentiated thyroid cancer; OS, overall survival.

Embolism was observed in four patients with DTC (pulmonary embolism, cerebral infarction, thrombotic cholecystitis, and deep vein thrombosis) and two patients with ATC (cerebral infarction and thrombotic cholecystitis). Gastrointestinal tract perforation was recognized in two patients with DTC. During treatment, irreversible regrowth occurred in seven patients with DTC who discontinued treatment because of this AE, and all these patients died. The AE characteristics are summarized in Table III.

OS curves. As shown in Fig. 3, 19 patients (including two overlapping cases) with serious AEs had poor outcomes, including a significantly lower $24-$ month OS rate $(\mathrm{P}<0.001)$. Meanwhile, the 24 -month OS rate was $73.2 \%$ in patients with

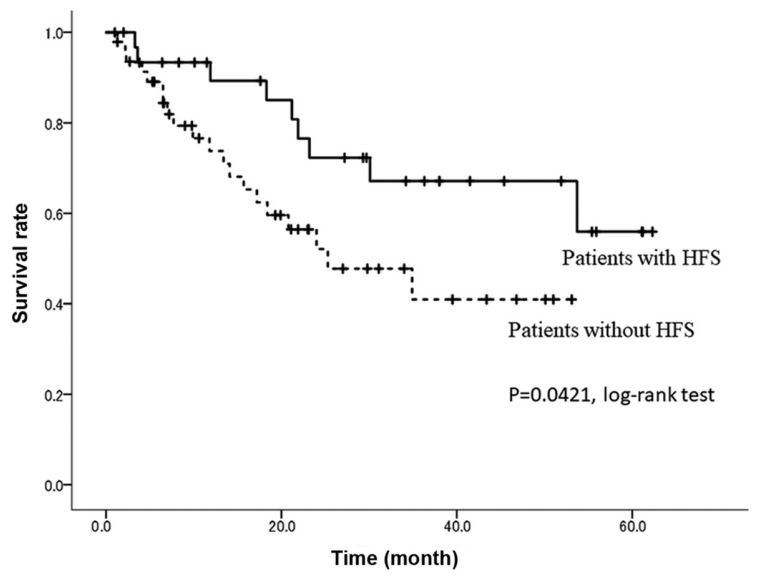

Figure 4. Comparison of overall survival curves for patients with and without HFS after lenvatinib treatment. The patients with HFS are represented by the bold line, and the patients without HFS are represented by the dotted line. The 24-month survival rate was $72.3 \%$ in patients with HFS, compared with $52.1 \%$ for patients without HFS $(\mathrm{P}=0.0421)$. HFS, hand-foot syndrome.

HFS compared with $52.1 \%$ in patients without HFS ( $\mathrm{P}=0.0421$, Fig. 4).

\section{Discussion}

We previously reported the results of lenvatinib for the treatment of ATC $(16,17)$. In this prior study, 19/32 lesions were diagnosed by biopsy only, and total thyroidectomy was not performed. Skin fistula was recognized in 11/19 patients (57.8\%). The oral treatment period was too short to consider other AEs. In many cases, even if AEs occurred, the disease progressed quickly, and the lenvatinib dose could not be reduced. With DTC, the administration period was long, and hence it was possible to evaluate AEs other than skin fistula. HT of $91.1 \%$, which was most common, did not correlate with prognosis because of its high frequency. Proteinuria and anorexia also appeared during the entire administration period, and no correlation with prognosis was observed. HT was the most common AE; nonetheless, HT of all patients was well controlled by administering 1-3 types of antihypertensive drugs and adjusting the dose. Regarding proteinuria, renal failure could be prevented by reducing the dose of lenvatinib and withdrawing this drug adequately (14). Meanwhile, anorexia and weight loss due to lenvatinib improved with drug suspension and resumption of dose reduction.

HT and HFS appeared within 1 month of administration in all cases, and a correlation with prognosis was observed in HFS. No correlation between HFS and prognosis was observed in prior studies.

In the real world, the lenvatinib therapy outcome for DTC was worse than that in the Select trial because of disease progression in the patients (18), in line with other reports (19). Of course, the management of non-serious AEs, such as hypertension, proteinuria, and HFS, is also necessary; however, as observed in the present study, prognosis will be poor if serious AEs are present. Therefore, careful management to avoid these AEs is essential to improve patient outcomes.

TKIs inhibit angiogenesis, and as such, careful administration is required because lesions adjacent to large blood vessels 
are at risk of bleeding (9). In particular, there is a risk of blood vessel wall rupture in lesions with a history of external irradiation (20) or for fistulae that develop in the digestive tract or skin (21). Although TKIs are not curative, they can be administered continuously. If a fistula develops, it is necessary to halt treatment or reduce the dose, whereas dose reduction may be preferable to treatment discontinuation to prevent irreversible tumor regrowth.

Anticoagulant therapy should be administered to patients at risk of embolism. For gastrointestinal perforation and cavity formation in the lungs, early detection, dose reduction, and drug interruption may be necessary for symptoms, such as abdominal pain and chest pain.

Avoiding serious AEs is paramount for improving the treatment outcomes. If a skin fistula develops, especially if it is close to a large blood vessel, the drug needs to be discontinued. Dose reduction is necessary to avoid fistula development in the digestive tract or trachea. Moreover, although embolism is difficult to prevent because of its sudden onset, d-dimer should be monitored in the high-risk groups, and anticoagulants should be administered as per requirement. Lenvatinib treatment has also been initiated for hepatic cancer; with an average dosing period of 6 months, the overall response and disease control rates were 57.1 and $71.4 \%$, respectively (22).

The frequency of HFS is high in Japan, as has been reported for several TKIs (13). Although the precise mechanisms by which multikinase inhibitors cause HFS remain unknown, the antiangiogenic activity of these drugs may inhibit vascular repair mechanisms in high-pressure areas, such as the palms and soles, which are repeatedly exposed to subclinical trauma (23).

This study was retrospective. Because the subjects were patients with ATC or DTC, which have different biological prognoses, the frequencies of AEs were also different. To examine whether the prognosis of DTC can be predicted by the presence or absence of HFS, a median treatment period of 18.2 months is not sufficient. Moreover, prognosis was significantly worse for patients with serious AEs.

This study, reported from real-world experience, showed that lenvatinib therapy could result in severe AEs requiring dose reduction or treatment discontinuation. It also indicated that the appearance of HFS portended a good prognosis in patients treated with lenvatinib, which may be characteristic of the Japanese population. We expect additional reports from other facilities regarding these two points.

\section{Acknowledgements}

The authors would like to thank Dr Hiroyuki Hayashi (Department of Pathology, Yokohama City Hospital, Yokohama, Japan) for assistance in pathological diagnosis.

\section{Funding}

No funding was received.

\section{Availability of data and materials}

All data generated or analyzed during this study are included in this published article.

\section{Authors' contributions}

HI and ST designed the study. HI, ST, SK and DM designed the study and investigation. SK and AM analyzed the data and contributed to data curation. All authors read and approved the final manuscript.

\section{Ethics approval and consent to participate}

The Cancer Board of Kanagawa Cancer Center Hospital (Japan) approved lenvatinib treatment, including surgery, for patients with ATC or DTC. The study was approved by the Institutional Review Board of Kanagawa Cancer Center Hospital (Japan). All patients provided a comprehensive consent stating that their samples that were collected for medical examination could be utilized for investigation and clinical research.

\section{Patient consent for publication}

All patients signed a consent form stating that their personal data and samples could be used for academic presentation or paper presentation while ensuring complete anonymity before receiving the treatment.

\section{Competing interests}

The authors declare that they have no competing interests.

\section{Authors' information}

$\mathrm{HI}$ is an endocrine surgeon working at Kanagawa Cancer Center and has extensive experience of several surgeries for ATC, as well as ATC treatment.

\section{References}

1. Seib CD and Sosa JA: Evolving understanding of the epidemiology of thyroid cancer. Endocrinol Metab Clin North Am 48: 23-35, 2019.

2. Olson E, Wintheiser G, Wolfe KM, Droessler J and Silberstein PT: Epidemiology of thyroid cancer: A review of the National Cancer Database, 2000-2013. Cureus 11: e4127, 2019.

3. Melosky B, Leighl NB, Rothenstein J, Sangha R, Stewart D and Papp K: Management of EGFR TKI-induced dermatologic adverse events. Curr Oncol 22: 123-132, 2015.

4. Eskazan AE and Ozmen D: Tyrosine kinase inhibitor (TKI) therapy for newly-diagnosed patients with chronic myeloid leukemia: Focusing on TKI discontinuation due to adverse events-is better always good? Expert Rev Hematol 10: 583-586, 2017.

5. Haugen BR: 2015 American Thyroid Association Management Guidelines for adult patients with thyroid nodules and differentiated thyroid cancer: What is new and what has changed? Cancer 123: 372-381, 2017.

6. Iwasaki H, Yamazaki H, Takasaki H, Suganuma N, Sakai R, Nakayama H, Hatori S, Toda S and Masudo K: Treatment outcomes of differentiated thyroid cancer with distant metastasis improve by tyrosine kinase inhibitors. Oncol Lett 17: 5292-5300, 2019.

7. Kamba T and McDonald DM: Mechanisms of adverse effects of anti-VEGF therapy for cancer. Br J Cancer 96: 1788-1795, 2007.

8. Sonpavde G, Bellmunt J, Schutz F and Choueiri TK: The double edged sword of bleeding and clotting from VEGF inhibition in renal cancer patients. Curr Oncol Rep 14: 295-306, 2012.

9. Machiels JP, Henry S, Zanetta S, Kaminsky MC, Michoux N, Rommel D, Schmitz S, Bompas E, Dillies AF, Faivre S, et al: Phase II study of sunitinib in recurrent or metastatic squamous cell carcinoma of the head and neck: GORTEC 2006-01. J Clin Oncol 28: 21-28, 2010. 
10. Mancuso MR, Davis R, Norberg SM, O'Brien S, Sennino B, Nakahara T, Yao VJ, Inai T, Brooks P, Freimark B, et al: Rapid vascular regrowth in tumors after reversal of VEGF inhibition. J Clin Invest 116: 2610-2621, 2006.

11. Liu L, Wang E, Li L, Chen D, Peng K, Wang M and Han G: As clinical markers, hand-foot-skin reaction and diarrhea can predict better outcomes for hepatocellular carcinoma patients receiving transarterial chemoembolization plus sorafenib. Can J Gastroenterol Hepatol 2019: 2576349, 2019.

12. Falcone G, Arrigoni C, Dellafiore F, Gallucci F, Milani V, Boveri S, Ausili D and Caruso R: A systematic review and meta-analysis on the association between hand-foot syndrome (HFS) and cancer chemotherapy efficacy. Clin Ter 170: e388-e395, 2019.

13. Kobayashi K, Kawakami K, Yokokawa T, Aoyama T, Suzuki K, Wakatsuki T, Suenaga M, Sato H, Sugiyama E, Yamaguchi K and Hama T: Association of hand-foot skin reaction with regorafenib efficacy in the treatment of metastatic colorectal cancer. Oncology 96: 200-206, 2019.

14. Iwasaki H, Yamazaki H, Takasaki H, Suganuma N, Sakai R, Nakayama H, Toda S and Masudo K: Renal dysfunction in patients with radioactive iodine-refractory thyroid cancer treated with tyrosine kinase inhibitors: A retrospective study. Medicine (Baltimore) 98: e17588, 2019.

15. Eisenhauer EA, Therasse P, Bogaerts J, Schwartz LH, Sargent D, Ford R, Dancey J, Arbuck S, Gwyther S, Mooney M, et al: New response evaluation criteria in solid tumours: Revised RECIST guideline (version 1.1). Eur J Cancer 45: 228-247, 2009.

16. Iwasaki H, Yamazaki H, Takasaki H, Suganuma N, Nakayama H, Toda $\mathrm{S}$ and Masudo K: Lenvatinib as a novel treatment for anaplastic thyroid cancer: A retrospective study. Oncol Lett 16: 7271-7277, 2018

17. Iwasaki H, Toda S, Suganuma N, Murayama D, Nakayama $H$ and Masudo K: Lenvatinib vs. palliative therapy for stage IVC anaplastic thyroid cancer. Mol Clin Oncol 12: 138-143, 2020.
18. Schlumberger M, Tahara M, Wirth LJ, Robinson B, Brose MS Elisei R, Habra MA, Newbold K, Shah MH, Hoff AO, et al: Lenvatinib versus placebo in radioiodine-refractory thyroid cancer. N Engl J Med 372: 621-630, 2015.

19. Locati LD, Piovesan A, Durante C, Bregni M, Castagna MG, Zovato S, Giusti M, Ibrahim T, Puxeddu E, Fedele G, et al: Real-world efficacy and safety of lenvatinib: Data from a compassionate use in the treatment of radioactive iodine-refractory differentiated thyroid cancer patients in Italy. Eur J Cancer 118: 35-40, 2019.

20. Hui EP, Ma BBY, King AD, Mo F, Chan SL, Kam MKM, Loong HH, Ahuja AT, Zee BCY and Chan ATC: Hemorrhagic complications in a phase II study of sunitinib in patients of nasopharyngeal carcinoma who has previously received high-dose radiation. Ann Oncol 22: 1280-1287, 2011.

21. Blevins DP, Dadu R, Hu M, Baik C, Balachandran D, Ross W, Gunn B and Cabanillas ME: Aerodigestive fistula formation as a rare side effect of antiangiogenic tyrosine kinase inhibitor therapy for thyroid cancer. Thyroid 24: 918-922, 2014.

22. Katsura Y, Takeda Y, Ohmura Y, Sakamoto T, Kawai K, Inatome J, Murakami K, Naito A, Kagawa Y, Masuzawa T, et al: Experience of treatment with lenvatinib in patients with advanced HCC-A in a single institution. Gan To Kagaku Ryoho 46: 2101-2103, 2019 (In Japanese).

23. Yada M, Masumoto A, Motomura K, Tajiri H, Morita Y, Suzuki H, Senju T and Koyanagi T: Indicators of sorafenib efficacy in patients with advanced hepatocellular carcinoma. World J Gastroenterol 20: 12581-12587, 2014.

This work is licensed under a Creative Commons Attribution-NonCommercial-NoDerivatives 4.0 International (CC BY-NC-ND 4.0) License. 